\title{
Measured wideband characteristics of indoor channels at centimetric and millimetric bands
}

\author{
Wei Fan ${ }^{*}$, Ines Carton, Jesper $\varnothing$. Nielsen, Kim Olesen and Gert F. Pedersen
}

\begin{abstract}
Accurate characterization of spatial multipath channels at millimeter wave bands has gained significant interest both in industry and academia. A channel measurement was conducted at three different frequency bands, i.e., $2-4$, $14-16$, and $28-30 \mathrm{GHz}$ in a line-of-sight (LOS) and an obstructed-LOS (O-LOS) scenarios in an empty room environment. A vector network analyzer connected to a virtual uniform circular array and to a rotational directional horn antenna was used in the measurements, respectively. Angle-of-arrivals and delay-of-arrivals of the multipath components were obtained from the measurements for the three frequency bands. Room electromagnetic properties for the three different frequencies at different propagation scenarios were investigated as well.
\end{abstract}

Keywords: Millimeter wave channel measurements, Spatio-temporal channel modeling, Room electromagnetics, Angle of arrival estimation

\section{Introduction}

The increasing demand for higher data rates has motivated research in millimeter wave frequency bands for $5 \mathrm{G}$ cellular systems. Millimeter wave bands offer huge free spectrum and allow the implementation of massive antenna arrays due to the small wavelength $[1,2]$. Though broadband wireless access technology local multipoint distribution service (LMDS) already exists at $28 \mathrm{GHz}$, it is still unused. This band has been considered as a potential candidate for 5G cellular frequency due to its low atmospheric absorption, and availability of high gain adaptive antennas [3-6]. Accurate characterization of their spatial multipath channel at millimeter wave bands has gained significant interest both in industry and academia, as it is important for system design and performance analysis of future millimeter wave communication systems $[7,8]$.

While significant studies of channel characteristics were carried out at $60 \mathrm{GHz}$ for indoor and short-range scenarios, only a few measurement campaigns were conducted at $28 \mathrm{GHz}[9,10]$. Various measurement results have been reported for the $28 \mathrm{GHz}$ band in the literature for urban

*Correspondence: wfa@es.aau.dk

Department of Electronic Systems, Faculty of Engineering and Science,

Aalborg University, Aalborg, Denmark scenarios, e.g., $28 \mathrm{GHz}$ penetration and reflection measurement in [1,3], $28 \mathrm{GHz}$ path loss and signal outage analysis in [1,11], $28 \mathrm{GHz}$ angle of arrival and angle of departure analysis in $[1,5]$, etc. Measurement results in an indoor building-scale environment based on a homemade synchronous channel sounder were reported in [6]. To the best knowledge of the authors, very few papers have experimentally investigated the channel characteristics at $30 \mathrm{GHz}$. Measured results of indoor propagation channels at $30 \mathrm{GHz}$ were reported in $[12,13]$, where the focus was only on path loss and small-scale fading characteristics.

Extensive work has been done for channel characterization in the literature at current cellular frequencies $[14,15]$ and other frequency bands, e.g., $5.8 \mathrm{GHz}$ in $[16,17]$. The existing work in the literature, however, are lacking in the analysis on the frequency-dependent channel behaviors. The frequency dependency factor is important, as the larger bandwidth, e.g., several $\mathrm{GHz}$ or subbands separated by $\mathrm{GHz}$, might be utilized for future wireless systems. It would be highly interesting to compare the channel characteristics at current cellular frequency bands and at millimeter wave bands in the same propagation scenarios.

Room electromagnetics is a simple model that considers a line-of-sight (LOS) component (if present) and an exponential power-delay profile (PDP) decaying with

\section{Springer}

C 2016 Fan et al. Open Access This article is distributed under the terms of the Creative Commons Attribution 4.0 International License (http://creativecommons.org/licenses/by/4.0/), which permits unrestricted use, distribution, and reproduction in any medium, provided you give appropriate credit to the original author(s) and the source, provide a link to the Creative Commons license, and indicate if changes were made. 
the so-called reverberation time parameter, depending only on the wall area, the volume of the room, and an absorption coefficient. Room electromagnetics theory is interesting, as it does not require complete knowledge of the propagation environment, and it offers an alternative description of diffuse scattering in a room using only simple parameters. Validation of room electromagnetic theory was investigated for cellular frequencies [18-20]. However, very few results are available in the analysis on frequency dependency of room electromagnetics.

In this paper, we investigate the spatial-temporal characteristics of LOS and obstructed-LOS (O-LOS) scenarios in an indoor environment at three different frequency bands, namely, $2-4,14-16$, and $28-30 \mathrm{GHz}$. Angleof-arrival and delay-of-arrival of the main paths and room electromagnetics are investigated for different frequency bands. Through the extensive measurements, we would like to investigate: (1) whether channel characteristics at different frequency bands are different in the same propagation scenario; (2) whether room electromagnetics theory holds at different frequency bands; and (3) whether diffuse scattering is important to model at millimeter wave frequency bands.

The main contributions of the paper are summarized as follows:

- A sounding system using a VNA coupled to a virtual uniform circular array (UCA) and to a rotational directional antenna to resolve multipath components in delay and spatial domains at different frequencies is described.

- Spatial-temporal characteristics of the indoor channels at different frequencies are compared in LOS and obstructed-LOS scenarios. The trajectory of multipath components in a room are identified by relating the multipath angle and delay information to the room geometry.

- Room electromagnetic parameters at different frequencies are compared in LOS and O-LOS scenarios.

- Spatial multipath components estimated with a virtual UCA are compared with results obtained with rotational directional antenna measurements.

Virtual arrays (via mechanical displacement of a single Biconical antenna) and rotation of this paper is organized as follows. Section 2 describes the indoorpropagation channels and the frequency beamforming technique used to estimate the spatial and temporal properties of multipath channels. Section 3 discusses the measurement setup. Section 4 summarizes the measurement results. Finally, section 5 concludes the paper.

\section{Indoor propagation channels}

To resolve the multipath components in space, two measurement systems are generally used. One is to steer a highly directional antenna to scan the channel impulse response (CIR) from each angular direction. This gives the angle information of the channel directly. However, the disadvantage is that the antenna pattern is embedded into the results. The other method is to use an (virtual) array. The angle information of the multipath components can be obtained via beamforming or high-resolution algorithms [21]. A uniform circular array (UCA) is preferable to a uniform linear array (ULA), as the ULA cannot distinguish the paths symmetric with respect to the array line and the beam pattern of the ULA array is not uniform around the azimuth angle.

For static indoor measurements, the measured CIR is essentially only one snapshot of an environment, i.e., only one temporal observation is available. The spatial method discussed in [22], e.g., the classical beamforming and high resolution algorithms like MUSIC cannot be directly applied, since the covariance matrix of the array outputs would be rank-deficient due to the limited temporal samples. Different algorithms were proposed to address this issue, e.g., a spatial smoothing technique in [21] and an iterative 2D Unitary ESPRIT method in [23] for virtual planar arrays. In this paper, we are interested in both delay and angle information of the multipath components. For the sake of simplicity, a conventional frequency beamforming algorithm is used [24]. Note that frequency beamforming algorithms were only used to estimate the angle and delay of the direct path between the transmitter $(\mathrm{Tx})$ and receiver $(\mathrm{Rx})$ for indoor ranging and localization purpose in [24].

Assume a UCA with $P$ array elements arranged uniformly around its perimeter of radius $r$, each with angle $\theta_{i}=2 \pi \cdot i / P$ with $i \in[0, P-1]$ as illustrated in Fig. 1 . Note that the distance between two consecutive array elements should not be larger than $\lambda / 2$ to avoid spatial aliasing effects [22]. Assume that the CIR between the Tx and the center of the UCA is composed by $K$ multipath plane waves. The channel frequency response is:

$$
H(f)=\sum_{k=0}^{K-1} \alpha_{k} \exp \left(-j 2 \pi f \tau_{k}\right)
$$

where $\alpha_{k}$ and $\tau_{k}$ represent the complex amplitude and delay of the $k$ th wave with $k \in[0, K-1]$. The $k$-th wave impinging with angle of arrival $\varphi_{k}$ arrives at the $i$-th element with a delay $\tau_{k_{i}}$ with respect to the UCA center:

$$
\tau_{k_{i}}=-\frac{r \cdot \cos \left(\theta_{i}-\varphi_{k}\right)}{c}
$$




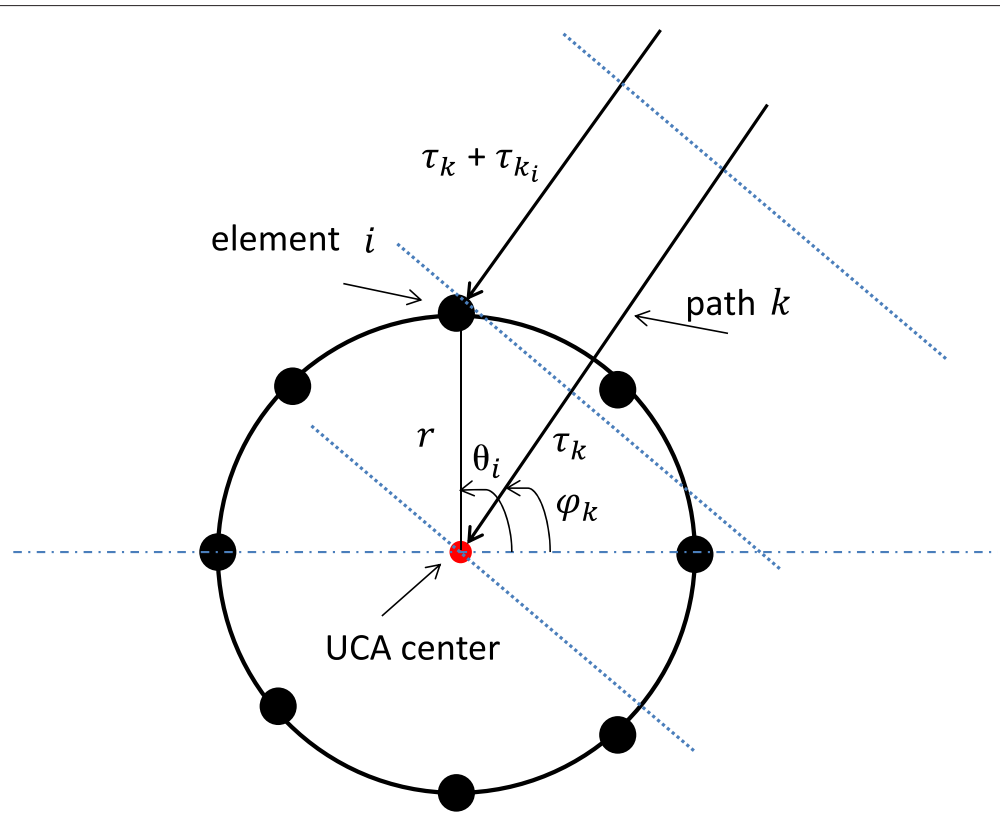

Fig. 1 An illustration of the UCA and the multipath channel

where $c$ is the speed of light. The frequency response $H_{i}(f)$ at the $i$ th element can be written as:

$$
H_{i}(f)=\sum_{k=0}^{K-1} \alpha_{k} \cdot \exp \left[-j 2 \pi f\left(\tau_{k}+\tau_{k_{i}}\right)\right]
$$

The array frequency response $H(f, \theta)$ is:

$$
H(f, \theta)=\frac{1}{P} \sum_{i=0}^{P-1} w_{i} H_{i}(f),
$$

where $w_{i}, i \in[0, P-1]$ is the complex weight assigned to the $i$ th antenna element. The basic principle of classical beamforming is to shift the phase of each frequency response into alignment. Therefore, the weighting vector can be expressed as:

$$
w_{i}=\exp \left(-j 2 \pi \cdot(r / c) \cdot \cos \left(\theta-\theta_{i}\right)\right),
$$

A power peak occurs in the array beam pattern for $\theta=$ $\varphi_{k}$. The spatial-temporal channel impulse response $h(t, \theta)$ can be recovered from $H(f, \theta)$ via inverse discrete Fourier transform:

$$
h(t, \theta)=\sum_{n=0}^{N-1} H\left(f_{n}, \theta\right) \cdot \exp \left(j 2 \pi f_{n} t\right),
$$

where $f_{n}$ is the frequency at the $n$-th frequency index.

\section{Experimental setup}

\subsection{Measurement system}

Figure 2 illustrates the block diagram of the measurement system. A wideband biconical antenna was mounted on a table as the transmitter (Tx), while two types of antennas were used as the receiver $(\mathrm{Rx})$. Note that both the Tx and Rx antenna were mounted at a height of $1.1 \mathrm{~m}$ above the ground. With the two different Rx antennas, two sets of measurements were performed. In the first measurements, a uniform circular array (UCA) was realized virtually by mounting a biconical antenna on a positioning turntable. We obtained $P=720$ elements of the UCA array by automatically repositioning the biconical antenna at uniform angles around a circle perimeter with $r=0.5 \mathrm{~m}$. At each element position, a frequency sweep was performed for three different frequency bands $2-4$, $14-16$, and $28-30 \mathrm{GHz} . N=750$ samples were set for each frequency band. In the second measurement series, a horn antenna was mounted on the rotation center of the turntable. To make meaningful comparison, the same measurement settings as in the first measurement series (i.e., the same frequency sweep and orientation sweep) were adopted for the second measurement series. A complete channel measurement, i.e., frequency sweep over the three bands for all element positions, took about $15 \mathrm{~min}$. Three types of antennas were used in the measurement campaign, as detailed in Table 1 . The biconical antenna is omnidirectional in the azimuth plane for $2-30 \mathrm{GHz}$, while the beamwidth gets narrower in the elevation plane as the operating frequency increases. The horn antenna operating at $750 \mathrm{MHz}-18 \mathrm{GHz}$ frequency band is less directional at the low band both in azimuth and elevation plane, and becomes more directional as the frequency increases. The horn antenna operating at $26.4-40 \mathrm{GHz}$ has approximately the same beamwidth in the elevation 


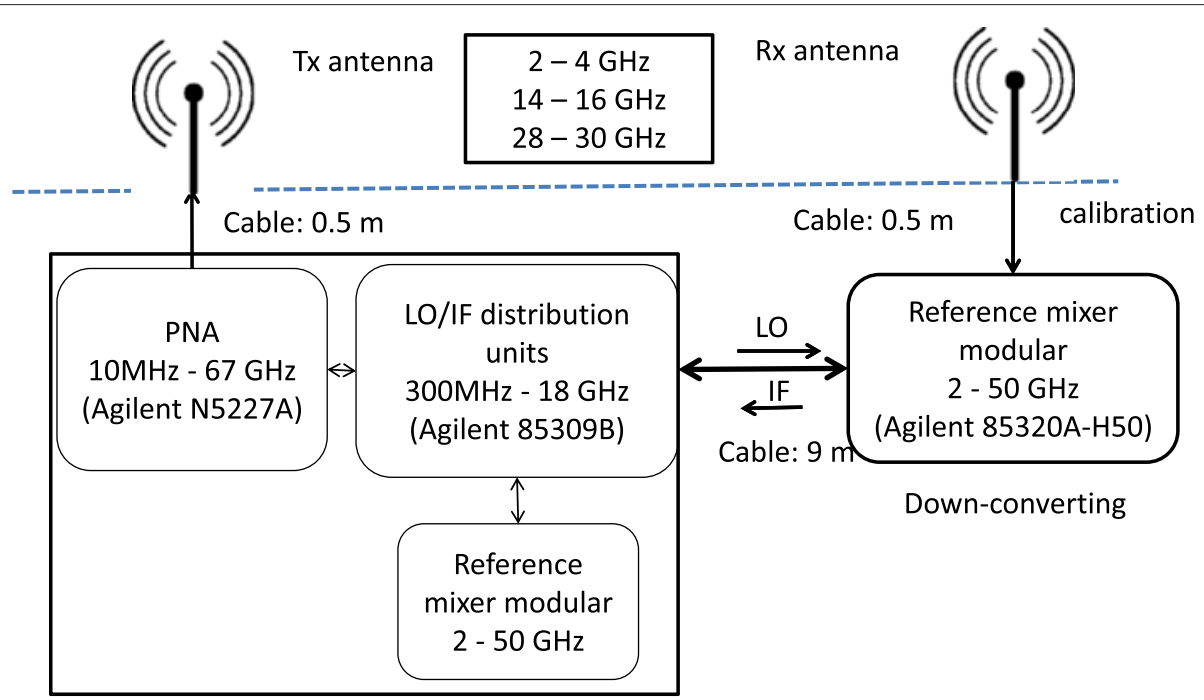

Fig. 2 Block diagram of the measurement system. The measurement system consists of a VNA, a LO/IF distribution unit, two reference mixer modular. The mixer modular is used to down-convert the received signals at the Rx to a lower frequency to reduce the cable loss and phase variation at the long cable $(9 \mathrm{~m})$. Calibration was performed prior to measurements to de-embed the Tx and Rx chain in the measurement results. Channel frequency responses at three frequency bands were recorded over one sweep

and azimuth plane. All the antennas used in the measurements are vertically polarized.

Given that the bandwidth of measurement at each frequency band is $2 \mathrm{GHz}$, the delay resolution for each band is $0.5 \mathrm{~ns}$, resulting in a spatial resolution is $0.15 \mathrm{~m}$. The delay range of the measurement is $350 \mathrm{~ns}$, which limits the maximum measurement range to approximately $105 \mathrm{~m}$ path length.

The measured $S_{i}^{21}(f)$ parameter can be expressed as a product of the Tx branch, Tx antenna, the channel, the Rx branch, and the Rx antenna. The Tx branch and the Rx branch could be removed via calibration, as illustrated in Fig. 2. The antenna gains of the Tx and Rx antenna at different frequency bands are given in Table 1, and can be de-embedded in post-processing to extract the channel frequency response.

\subsection{Measurement scenario}

The measurements were performed in an empty room, as depicted in Fig. 3. All walls are made of concrete except the bottom wall which is a wooden board as indicated in the figure. Two scenarios were considered in the measurement: LOS and O-LOS, as shown in Figs. 4 and 5, respectively. For the O-LOS measurement, a blackboard of dimensions $1.19 \times 1.19 \mathrm{~m}^{2}$ with one side covered by aluminum was placed between the antennas to block the paths in the LOS direction.

\subsection{Measurement objectives}

Five objectives were targeted in the measurement campaign:

1. In this paper, virtual arrays (via mechanical displacement of a single Biconical antenna) and rotation of high directive horn antennas are used to investigate the power-angle-delay profiles of the indoor propagation channels. To achieve this goal, the propagation environment should be maintained static during measurement. System stability investigation was carried out in Section 4.1.

2. Accurate calibration of antenna element positions is required, which becomes even more challenging at

Table 1 Specifications of the antennas used in the measurement campaign

\begin{tabular}{llll}
\hline Antenna & Frequency range & 3-dB beam width in azimuth & Gain \\
\hline Biconical antenna & $2-30 \mathrm{GHz}$ & Omnidirectional & $2-4 \mathrm{GHz}: 0 \mathrm{~dB}$ \\
& & & $14-16 \mathrm{GHz}: 5.5 \mathrm{~dB}$ \\
& & $28-30 \mathrm{GHz}: 6 \mathrm{~dB}$ \\
Horn antenna & $750 \mathrm{MHz}-18 \mathrm{GHz}$ & $2-4 \mathrm{GHz}: 65^{\circ}$ & $2-4 \mathrm{GHz}: 9 \mathrm{~dB}$ \\
& & $14-16 \mathrm{GHz}: 30^{\circ}$ & $14-16 \mathrm{GHz}: 15 \mathrm{~dB}$ \\
Horn antenna & $26.4-40.1 \mathrm{GHz}$ & $28-30 \mathrm{GHz}: 20^{\circ}$ & $28-30 \mathrm{GHz}: 19 \mathrm{~dB}$ \\
\hline
\end{tabular}




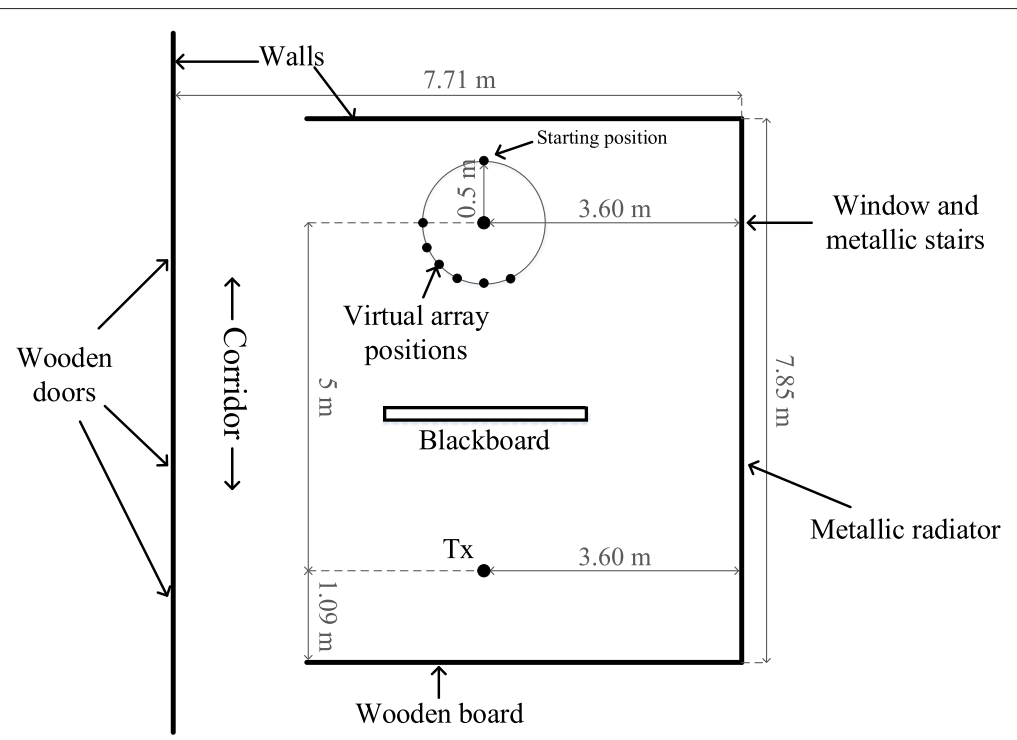

Fig. 3 An illustration of the dimensions of the empty room and locations for the Tx and Rx antennas for the measurement campaign

millimeter wave bands. For this reason, the measurements were repeated two to three times to investigate the repeatability of the results.

3. Wideband channel characteristics in delay and spatial domains and room electromagnetic in the LOS and O-LOS scenarios are analyzed and compared.

4. One interesting research topic in millimeter wave channel modeling is the differences and similarities in channel characteristics at different frequency bands. In this paper, measured channel characteristics at $2-4,14-16$, and $28-30 \mathrm{GHz}$ are compared.

5. Measurement results with a virtual UCA and steering horn antennas are analyzed and compared. Note that for the measurements with horns, two different horn antennas were used to cover the three frequency bands (one for $2-4$ and $14-16 \mathrm{GHz}$, and the other one for $28-30 \mathrm{GHz}$ ), as detailed in Table 1 .

Table 2 summarizes the measurement items performed in the measurement campaign.

\section{Measurement results}

\subsection{System stability}

To investigate the channel spatial profile by using a virtual array or rotating a high-directive horn antenna, it is important to ensure a static environment [16]. In order to investigate the stability of the measurement system, wideband measurements with a biconical antenna at the $\mathrm{Rx}$ side are performed, without rotating the turntable. That is, the channel frequency responses were repeated $P=$

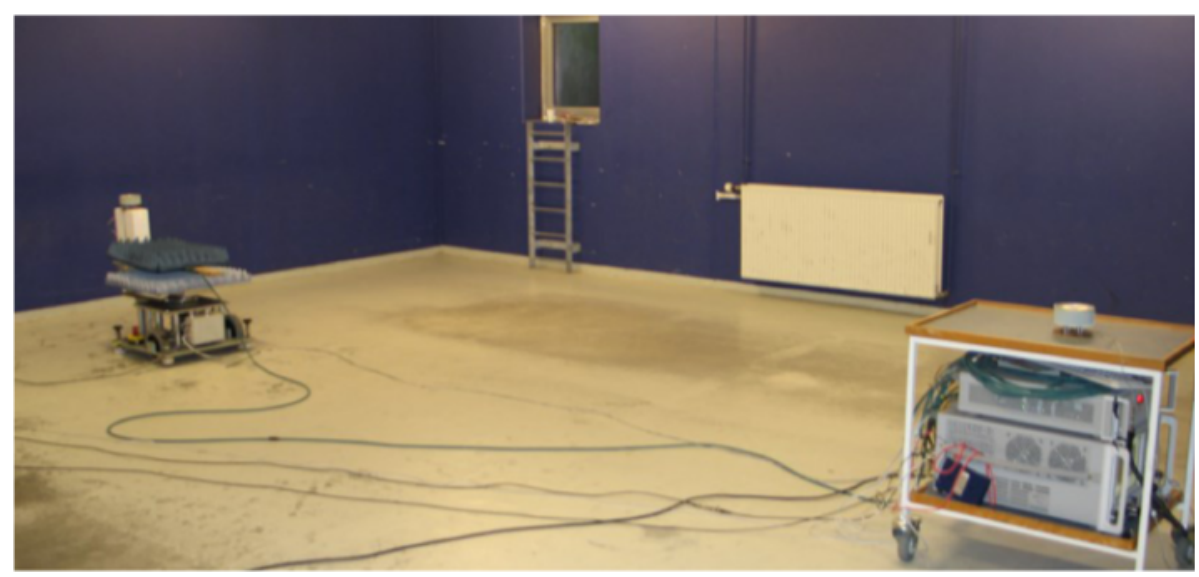

Fig. 4 Photograph of the line-of-sight (LOS) scenario 


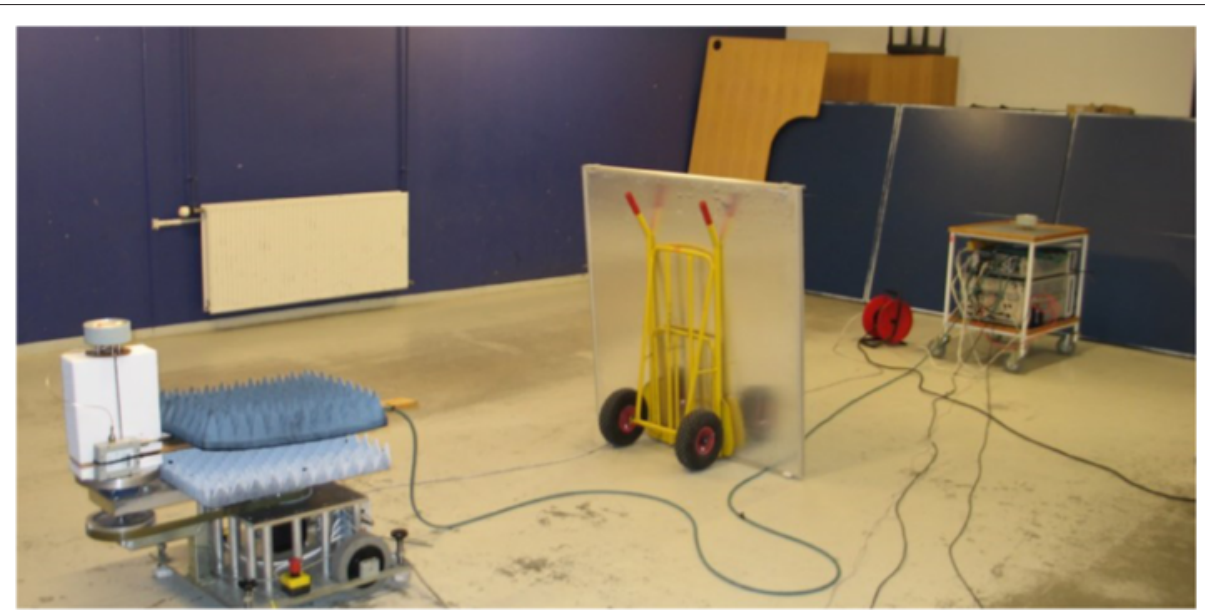

Fig. 5 Photograph of the O-LOS scenario

720 times for the same position of the Rx. CIRs can be calculated via inverse Fourier transform of the measured frequency responses for each frequency band. One simple way to check stability is to investigate the phase variation of the narrowband received signal, which is obtained by summing up the CIRs coherently over the delay domain. The rms phase variation of the received signal is shown in Table 3. The rms phase variation at $2-4 \mathrm{GHz}$ is up to $2.9^{\circ}$, which is higher compared to phase variation at $14-16$ and $28-30-\mathrm{GHz}$ bands both for the LOS and O-LOS scenarios. This might be caused by higher instabilities in the measurements system at lower frequencies. The rms phase variations are comparable in LOS and $\mathrm{O}$-LOS scenarios at all frequency bands.

\subsection{Repeatability}

Measurements with the horn antennas and the UCA were repeated to investigate the repeatability of the measurements. Note that in this case, the turntable is rotating as shown in Table 2 . To investigate the repeatability of the results, the cross correlations of the narowband received signals are calculated for each scenario among different repetitions. The cross correlation is equal to 1 when two measurement results are identical. The worst results among the repetitions are shown in Fig. 6. The

Table 2 Measured items during the measurement campaign

\begin{tabular}{llll}
\hline Item & Scenario & Repetition & Turntable \\
\hline Stability investigation & LOS & 1 & Static \\
\multirow{3}{*}{ UCA } & O-LOS & 1 & Static \\
& LOS & 3 & Rotation \\
Horn & O-LOS & 3 & Rotation \\
& LOS & 2 & Rotation \\
& O-LOS & 2 & Rotation \\
\hline
\end{tabular}

measurements are generally highly repeatable, with cross correlations between repetitions above 0.98 for all scenarios. The measurements with the horn antennas are less repeatable due to a stronger cable effect (e.g., cable bending) observed in practical measurements.

\subsection{Horn antenna results}

\subsubsection{Main path identification}

The measured power-angle-delay profiles with horn antennas for the LOS and O-LOS scenarios for different frequency bands are shown in Figs. 7 and 8, respectively. The trajectories of the multipath components in the measurement room can be obtained by relating the angle and delay information of the multipaths (Figs. 7 and 8), to the room geometry (Fig. 3), as shown in Fig. 9.

Note that the delay range is limited to $60 \mathrm{~ns}$ (corresponding to $18 \mathrm{~m}$ in distance) in Figs. 7 and 8 for illustration of the main paths. The spatial resolution is limited by the beamwidth of the horn antennas. As detailed in Table 1, the horn antenna operated at $2-4 \mathrm{GHz}$ suffers from low spatial resolution both in azimuth and elevation domains, while the horn antennas operated at $14-16$ and $28-30 \mathrm{GHz}$ are much more directional, with a beamwidth of $20^{\circ}$ at the $28-30 \mathrm{GHz}$ band in azimuth domains. The delay resolution, however, is the same, since the same bandwidth is set for the three frequency bands.

Table 3 rms phase variations of the received field unit [ $\left.{ }^{\circ}\right]$

\begin{tabular}{lll}
\hline & \multicolumn{2}{c}{ Static } \\
\hline Freq. [GHz] & LOS & O-LOS \\
$2-4$ & 2.4 & 2.9 \\
$14-16$ & 0.2 & 0.47 \\
$28-30$ & 0.7 & 1.5 \\
\hline
\end{tabular}




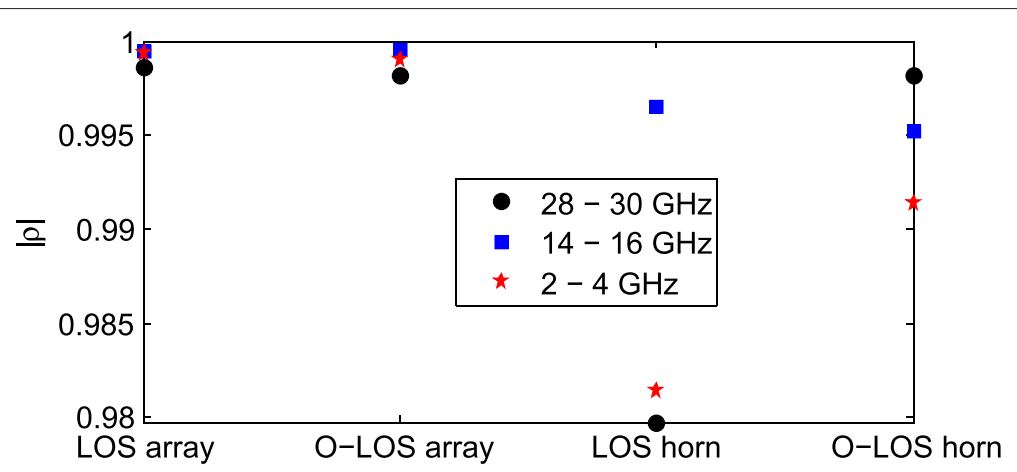

Fig. 6 Cross correlations of the received fields in two repeated measurements for different scenarios

LOS measurements at different frequency bands are dominated by the LOS paths, while the O-LOS measurement is more spatially rich, with quite a few dominant paths in various specular directions. As seen in Figs. 7 and 8, multipath components identified in the LOS and O-LOS scenarios are quite similar, except the paths impinging the $\mathrm{Rx}$ with AoAs around $0^{\circ}$ and $180^{\circ}$, which are blocked by the blackboard. It is worth noting that these paths have a slightly different interpretation in the LOS and O-LOS cases. For example, the path identified as 1
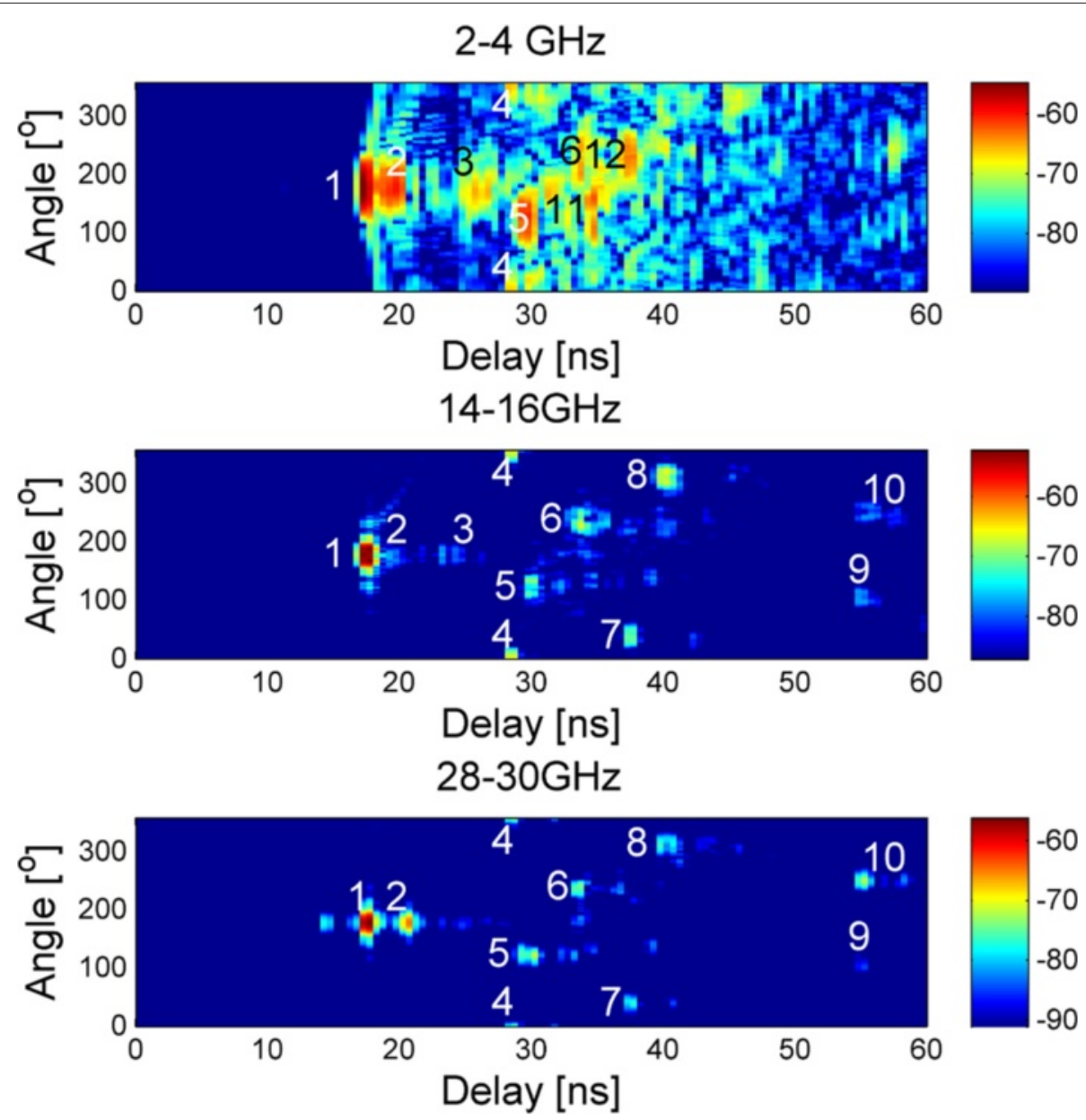

Fig. 7 Power-angle-delay profile measured with horn antennas for LOS scenarios at different frequency bands. The power dynamic range (i.e., color bar range) is limited to $35 \mathrm{~dB}$. Note that the horn antenna gain is embedded in the results for all the bands 

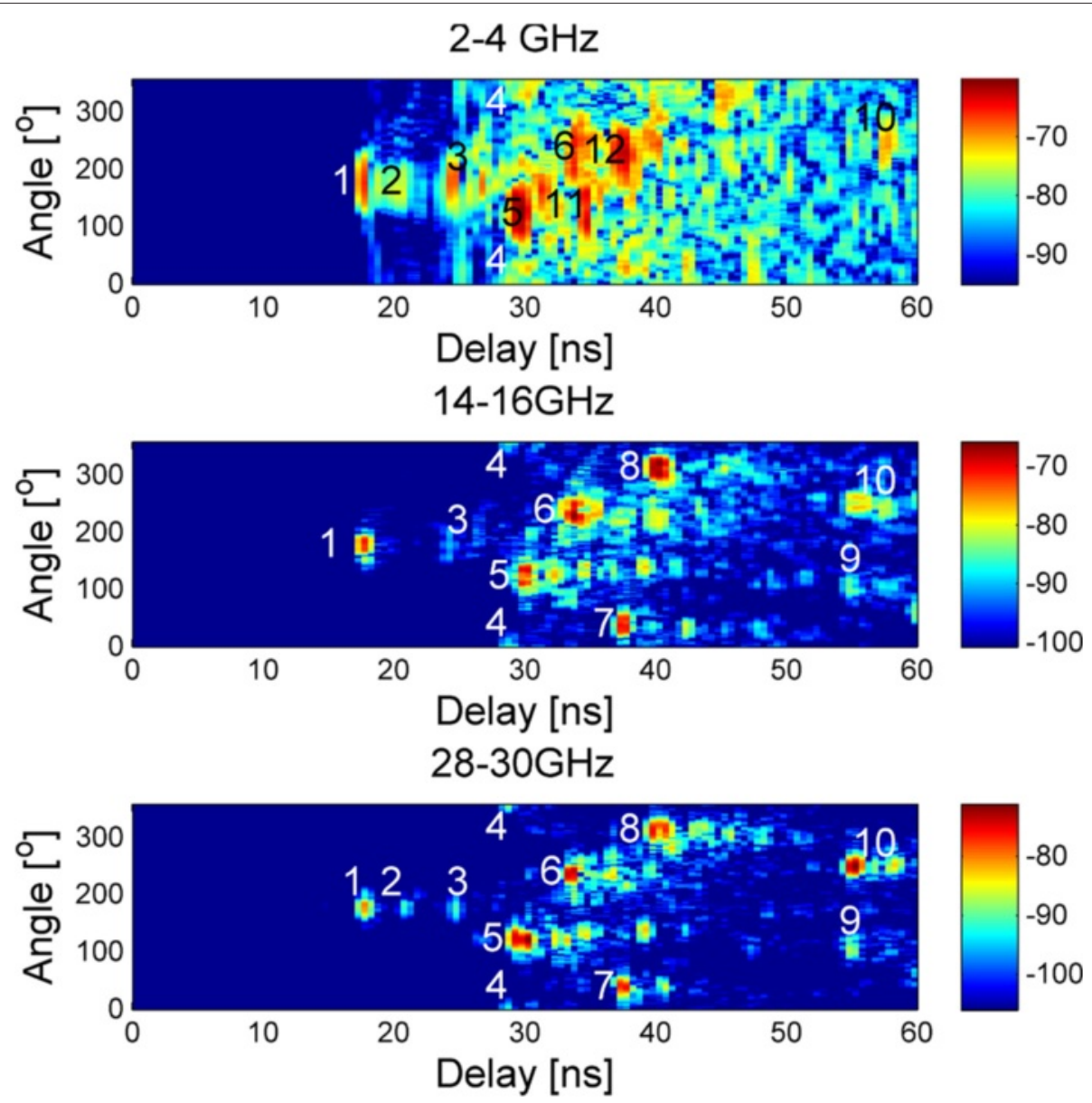

Fig. 8 Power-angle-delay profile measured with horn antennas for O-LOS scenarios at different frequency bands. The power dynamic range (i.e. color bar range) is limited to $35 \mathrm{~dB}$. Note that the horn antenna gain is embedded in the results for all the bands

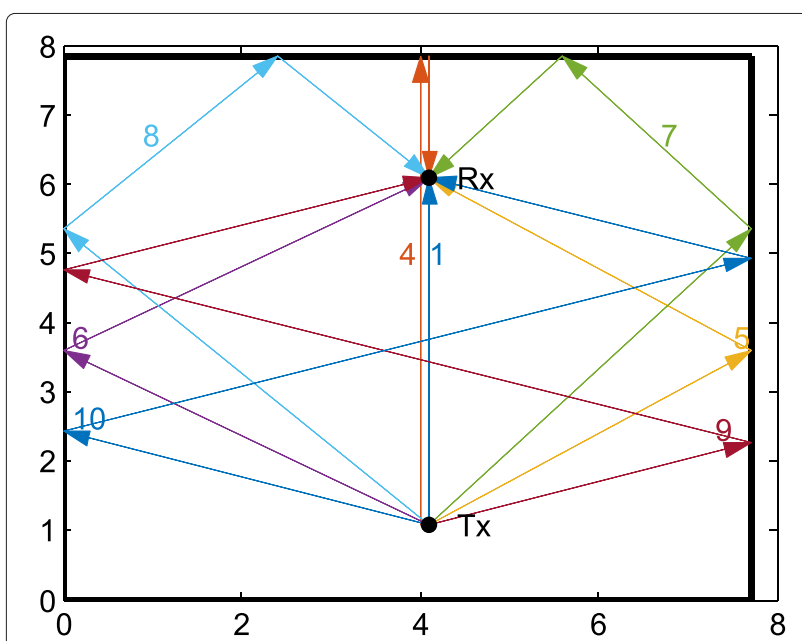

Fig. 9 Paths identified (top view) based on the results reported at $14-16 \mathrm{GHz}$ and $28-30 \mathrm{GHz}$ in Figs. 7 and 8 for the LOS and O-LOS scenarios. Note that path 2, which corresponds to a ground reflection, and path 3, which corresponds to a ceiling reflection, are not present in the figure has the same AoA for both cases, yet it corresponds to the LOS in Fig. 7, whereas in Fig. 8 it corresponds to a diffracted path from the top edge of the obstructor. The radius of the $n$th Fresnel zone can be calculated as:

$$
F_{n}=\sqrt{\frac{n \lambda d_{1} d_{2}}{d_{1}+d_{2}}},
$$

where $d_{1}, d_{2}$ is the distance from the obstructor to the $\mathrm{Tx}$ and $\mathrm{Rx}$, respectively. In our measurement, the radius of the first Fresnel zone at $30 \mathrm{GHz}$ is $0.11 \mathrm{~m}$, whereas the height difference between the blackboard and the antennas is only $0.09 \mathrm{~m}$; thus the first Fresnel zone was not completely obstructed by the blackboard, resulting in the diffraction identified as path 1 in Fig. 8. The power angle spectra measured for the LOS and O-LOS scenarios at $14-16$ and $28-30-\mathrm{GHz}$ frequency bands are shown in Fig. 10. The power angle spectra in the LOS scenario match quite well with those in the O-LOS scenario in non-LOS directions for both frequency bands, as expected. 


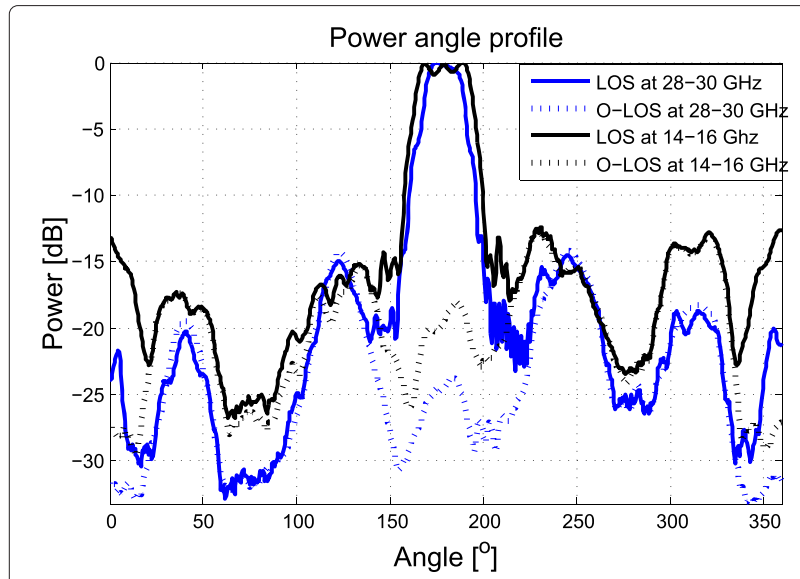

Fig. 10 Power angle spectra measured for the LOS and O-LOS scenarios at $14-16$ and $28-30-\mathrm{GHz}$ frequency bands. Note the power values at two different bands are normalized to the LOS component at $14-16$ and $28-30 \mathrm{GHz}$, respectively

Contrary to the fact that only a few specular paths are identified at $14-16$ and $28-30 \mathrm{GHz}$, measured channels at $2-4 \mathrm{GHz}$ present quite different results. This observation is consistent with results published in the literature, i.e., channel profiles at millimeter wave bands are characterized by fewer and sparser dominant paths compared to typical cellular bands $[9,10]$. A richer multipath environment can be observed for the results at $2-4 \mathrm{GHz}$, with many more paths present in the delay domain. This might be due to a stronger diffuse scattering from objects at the low frequency band, as the wavelength is more comparable to object dimensions at the low frequency. At the higher bands, the wavelength is much smaller, and hence specular reflections are dominant. Different dominant paths can be identified at $2-4 \mathrm{GHz}$. Although some specular paths can be identified at all frequencies (e.g., path $1,2,3,4,5$, and 6 ), some of the paths present at $2-4$ $\mathrm{GHz}$ can not be identified at higher frequency bands (e.g., path 11 and 12). Also, some specular paths present at high bands (e.g., path 7 and 8) cannot be clearly identified at the low frequency band.

The main propagation paths from the Tx to the Rx can be identified, as shown in Fig. 9. The angles and delays of the identified paths at $14-16$ and $28-30 \mathrm{GHz}$ are quite similar both for the LOS and O-LOS scenarios. However, the normalized power values of the identified paths with same delay and angle at different frequency bands might be different. As shown in Fig. 10, although main paths impinge the $R x$ at same angles, the power values are different.

In [2], scattering effects are shown in the measurements, whereas only specular components can be seen in our measurements at $28-30 \mathrm{GHz}$. This might be due to the fact that a small furnished office, where small scatterers exist, was investigated in [2], whereas an empty room with a few scatterers was selected in this paper. Moreover, a wide dynamic range was used in [2], whereas in this paper, the dynamic range in this paper is limited to $35 \mathrm{~dB}$ in order to investigate only the dominant components. A richer multipath environment could be expected in a furnished room as shown in [16].

\subsubsection{Room electromagnetic}

Discussions in section 4.3.1 were focused on the accurate characterizations of the main propagation paths, i.e., the LOS path and main reflected paths from the walls and ground. We are also interested in the remaining part of the CIRs that correspond to the non-dominant paths. The rms delay spread $\tau_{\text {rms }}$ and mean excess delay $\bar{\tau}$ are often calculated with respect to a threshold relative to the strongest path. This ensures full compatibility of the results, since noise is excluded from the calculation. The $\tau_{\text {rms }}$ and $\bar{\tau}$ using a $30 \mathrm{~dB}$ threshold are shown in Table 4 for the LOS and O-LOS scenarios at different frequency bands. $\tau_{\text {rms }}$ and $\bar{\tau}$ are larger in the O-LOS scenarios compared with the LOS scenarios for all frequency bands as expected, with an exception that $\tau_{\text {rms }}$ in the O-LOS scenario is slightly smaller at $2-4 \mathrm{GHz}$, compared to the higher frequency bands. $\tau_{\text {rms }}$ and $\bar{\tau}$ at $2-4 \mathrm{GHz}$ in the LOS scenario are larger compared to higher frequencies due to the richer multipath environments at the low band. $\tau_{\text {rms }}$ and $\bar{\tau}$ at the O-LOS scenarios are, however, comparable at the three different frequency bands.

The tail of the power delay profiles (PDPs) generally have exponential decays with approximately the same decay rates for different scenarios at different frequency bands, as shown in Table 4, with a deviation up to 0.02 $\mathrm{dB} / \mathrm{ns}$ around $-0.26 \mathrm{~dB} / \mathrm{ns}$ decay rate. The decay rate of the delay tail is governed by the overall dimensions of the room and an average absorption coefficient, and it is irrelevant to the propagation scenarios (i.e., LOS or O-LOS) [18]. This is due to the fact that the room will be filled with energy, resulting in a constant energy density for the same delay [18]. It can be concluded based on the measurement results that the average absorption coefficient does not depend on frequency. Note that the decay rates were calculated based on CIRs ranged from 60 to $200 \mathrm{~ns}$ to avoid including the dominant paths. If the CIRs before $60 \mathrm{~ns}$, where dominant paths are present, are considered, the calculated decay rate will be different. The PDPs for

Table $4 \tau_{\text {rms }}$ and $\bar{\tau}$ for the LOS and O-LOS scenarios at different frequency bands

\begin{tabular}{llllllll}
\hline & \multicolumn{3}{c}{ LOS } & \multicolumn{3}{c}{ O-LOS } \\
\hline Freq. [GHz] & $2-4$ & $14-16$ & $28-30$ & $2-4$ & $14-16$ & $28-30$ \\
$\bar{\tau}$ [ns] & 29.5 & 20.2 & 19.5 & 37.7 & 38.8 & 37.1 \\
$\tau_{\text {rms }}[\mathrm{ns}]$ & 12.9 & 7.3 & 6.1 & 12.6 & 10.9 & 11.0 \\
Rate [dB/ns] & -0.25 & -0.25 & -0.26 & -0.24 & -0.27 & -0.27 \\
\hline
\end{tabular}




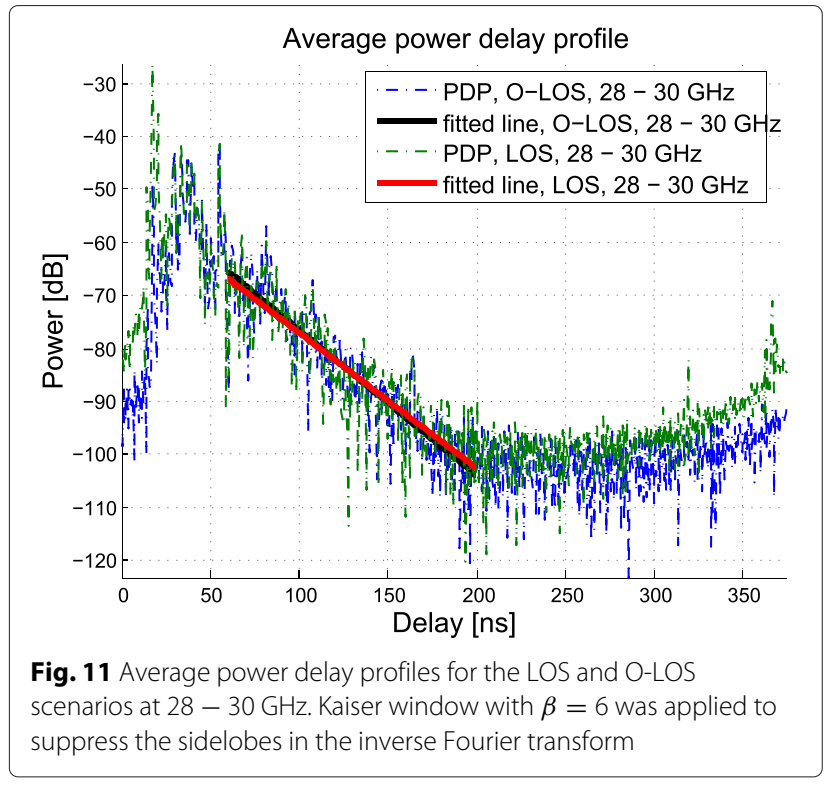

the LOS and O-LOS scenarios at $28-30 \mathrm{GHz}$ and the fitted lines are shown in Fig. 11. Note that different scenarios at different frequencies not only have the delay tails with the same decay rates, but also the power values in tails are the same as well, as shown in Fig. 11.

\subsection{UCA measurement results}

4.4.1 Path loss

For the virtual UCA measurements, the path loss at each element position $i$ at each frequency band can be expressed as:

$$
P L(i)=\frac{1}{N} \sum_{n=1}^{N}\left|H_{i}\left(f_{n}\right)\right|^{2} .
$$

The path loss results for the LOS and O-LOS scenarios for the three frequency bands are shown in Fig. 12. In the LOS scenario, the measured path loss results match well with the calculated path loss from Friis equation, as the LOS path is dominant. The deviation is mainly due to the inaccurate antenna gains provided in Table 1 for different frequency bands. The ripples in the measured results are caused by the paths from non-LOS directions. The path loss in the LOS scenario varies along element positions as expected, e.g., minimum path loss present at element position 360 (i.e., orientation angle $180^{\circ}$ ), which corresponds well to the element positions illustrated in Fig. 3. The path loss in O-LOS scenario is around 10 $\mathrm{dB}$ higher compared to LOS scenario for $14-16$ and $28-30-\mathrm{GHz}$ bands, while only around $4 \mathrm{~dB}$ higher for $2-4 \mathrm{GHz}$.

\subsubsection{Power-angle-delay profile}

The CIRs received at different UCA element positions for the LOS scenario at different frequency bands are shown in Fig. 13. Similar to horn antenna measurement results, a richer multipath environment can be observed at $2-4$ $\mathrm{GHz}$, compared with higher frequency bands. Only a few specular paths can be identified from the delay variation along array element positions at $14-16$ and $28-30 \mathrm{GHz}$.

The power-angle-delay profiles obtained with frequency beamforming techniques discussed in Section 2 for the LOS and O-LOS scenarios at different frequency bands are shown in Figs. 14 and 15, respectively. Similar to the horn antenna measurement results, richer multipath environments can be observed at $2-4 \mathrm{GHz}$, compared to results in high frequency bands, both for the LOS and O-LOS scenarios. Similar power-angle-delay profiles are present at $14-16$ and $28-30-\mathrm{GHz}$ frequency bands. A few dominant specular paths can be identified for all
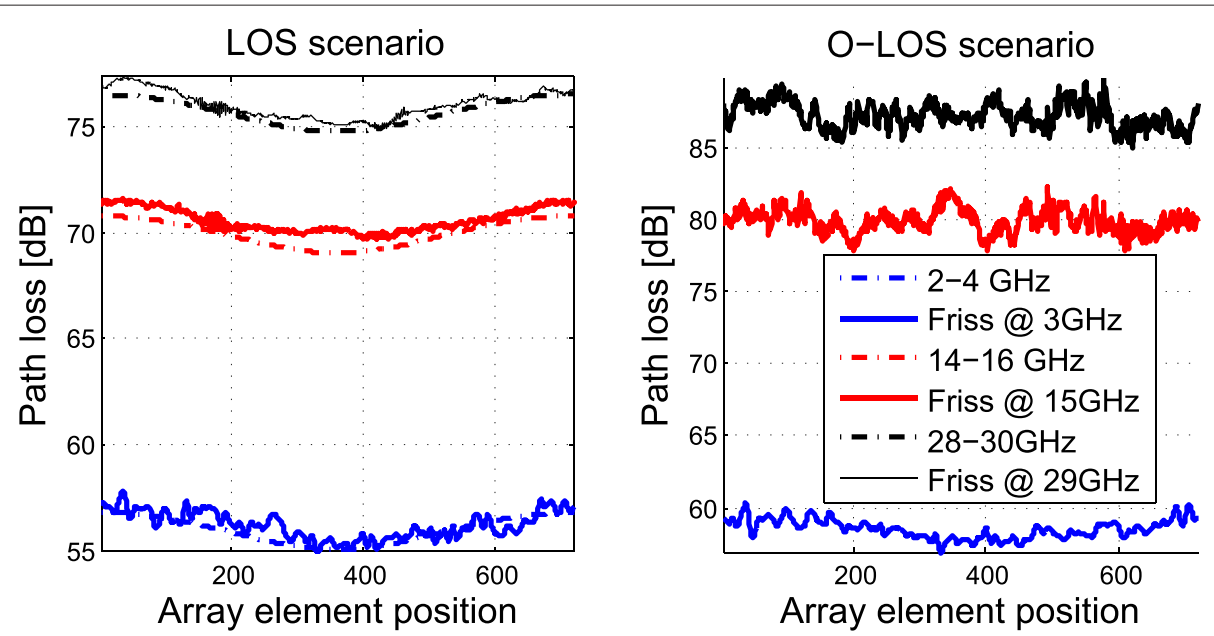

Fig. 12 Path loss over different array element positions for three frequency bands for the LOS and O-LOS scenarios. Note that the transmit and receive antenna gains at different frequency bands are de-embeded from the results 

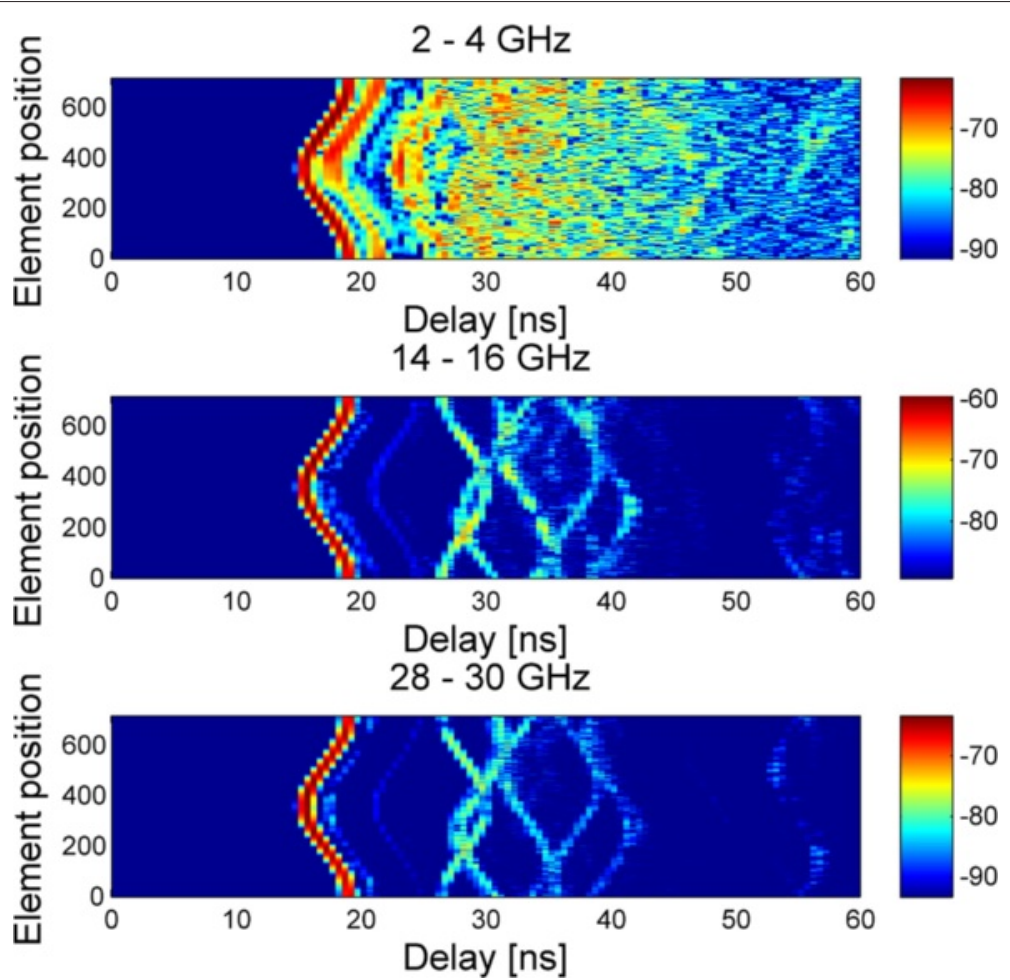

Fig. 13 CIRs recorded at different UCA element positions for the LOS scenario at different frequency bands
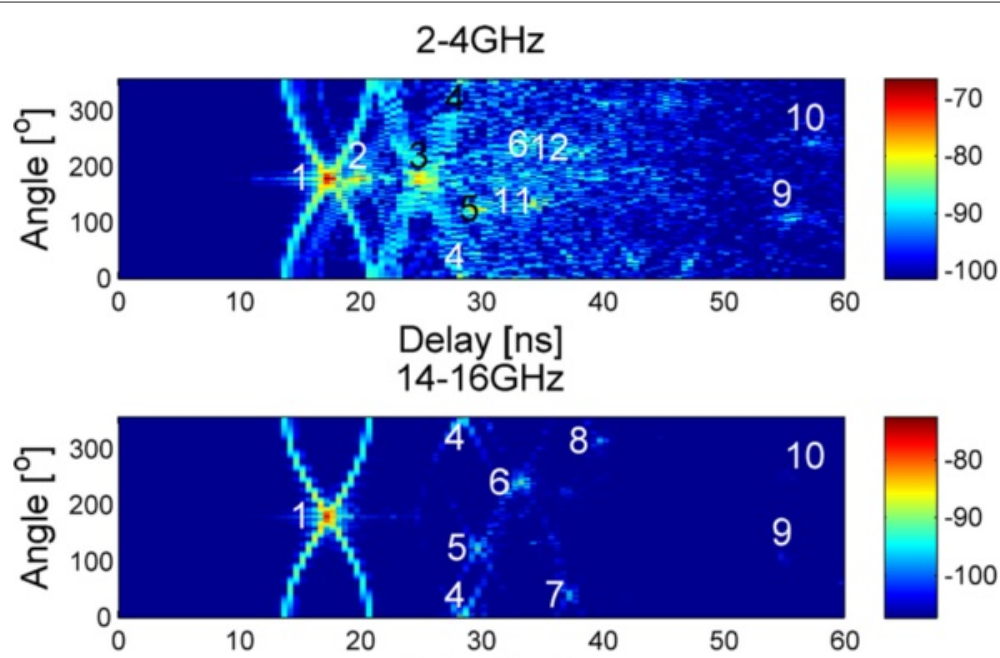

Delay [ns]

28-30GHz

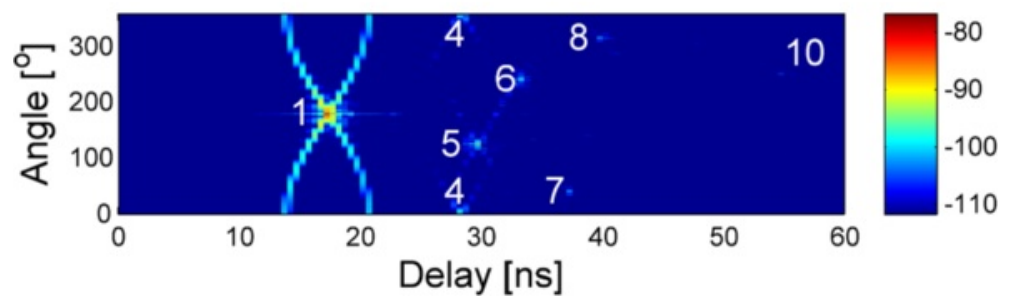

Fig. 14 Power-angle-delay profile measured with UCA for LOS scenarios at different frequency bands. The power dynamic range is limited to $35 \mathrm{~dB}$ 

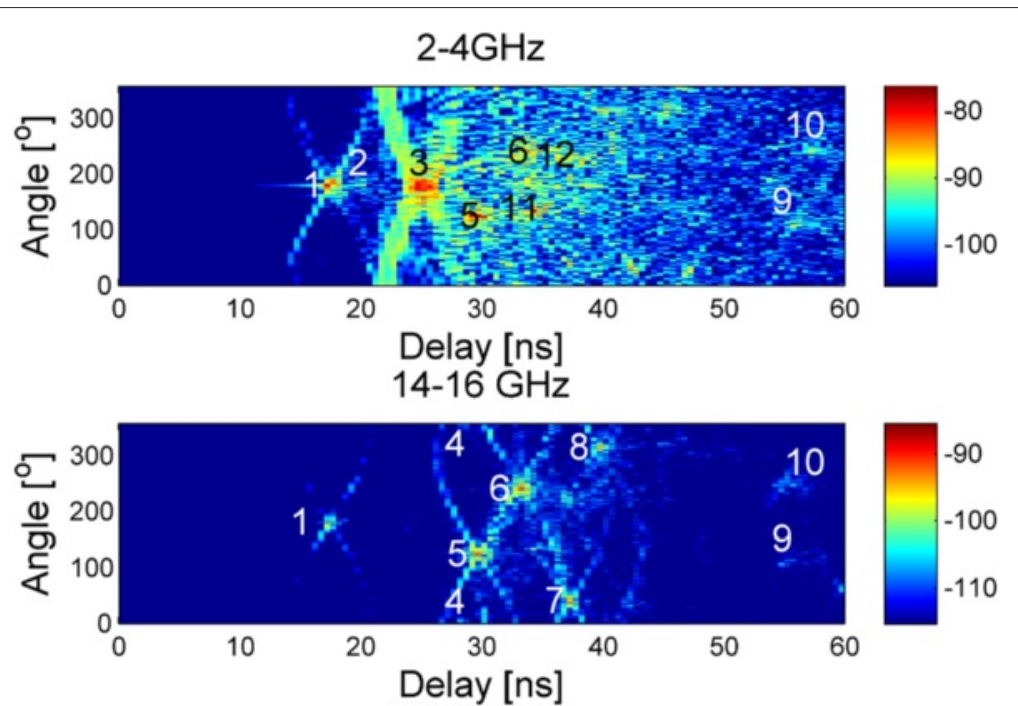

28-30GHz

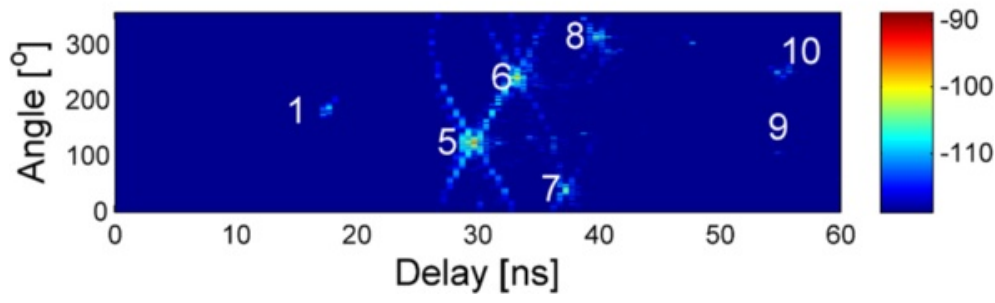

Fig. 15 Power-angle-delay profile measured with UCA for O-LOS scenarios at different frequency bands. The power dynamic range is limited to $30 \mathrm{~dB}$

the frequency bands. Compared with horn antenna results in Figs. 7 and 8, paths with same impinging angles and delays are observed both for the LOS and O-LOS scenarios at different the frequency bands. The measured power-angle-delay profiles with the UCA, although with higher spatial resolution, suffers from high side lobes as well. As explained in [24], the joint sidelobes in delay and angle domains are caused by the joint frequency and angle dependance inherent in phase in Eq. (4) with the conventional beamforming technique. The focus of future work will be on resolving the multipath components from the array measurement results with other techniques, e.g., frequency invariant beamforming technique [24].

\section{Conclusions}

This work presents an extensive measurement campaign and a detailed analysis of spatial-temporal channels at centimetric and millimetric bands in an indoor environment. Multipath components were resolved in space and delay via steering a wideband horn antenna and via measurements with a virtual wideband UCA in different frequency bands, respectively. Measurement results include stability levels, repetition levels, powerangle-delay profiles, path loss, room electromagnetic, etc. Results show that:
- The measurement system is quite stable, with a rms phase drift up to $1.5^{\circ}$ at $28-30 \mathrm{GHz}$ band.

- The measurement results are highly repeatable, with a correlation between two repeated measurements up to 0.98 .

- Richer multipath components are present at lower frequency bands due to diffuse scattering effects, while specular reflections are dominant at millimeter wave bands. The trajectory of the specular components in a room are identified by relating the multipath angle and delay information to the room geometry. Investigations in a furnished room, where more scatterers exists, will be performed in future work.

- Room electromagnetics are not frequency dependent in an empty indoor scenario. The tails of the PDPs can be modeled with a exponential decay with decay rate around $-0.25 \mathrm{~dB} / \mathrm{ns}$.

\section{Competing interests}

The authors declare that they have no competing interests.

\section{Acknowledgements}

This work has been supported by the Danish High Technology Foundation via the VIRTUOSO project. The authors appreciate the assistance from Kristian Bank and Yi Tan with the practical measurements. The authors would like to thank Dr. Lishuai Jing and Dr. Ming Shen for the active discussions. 
Received: 9 June 2015 Accepted: 4 February 2016

Published online: 20 February 2016

\section{References}

1. T Rappaport, S Sun, R Mayzus, H Zhao, Y Azar, K Wang, G Wong, J Schulz, M Samimi, F Gutierrez, Millimeter wave mobile communications for $5 \mathrm{G}$ cellular: It Will Work!. Access. IEEE. 1, 335-349 (2013)

2. V Degli-Esposti, F Fuschini, E Vitucci, M Barbiroli, M Zoli, L Tian, X Yin, D Dupleich, R Muller, C Schneider, R Thoma, Ray-tracing-based mm-wave beamforming assessment, Access. IEEE. 2, 1314-1325 (2014)

3. H Zhao, R Mayzus, S Sun, M Samimi, I Schulz, Y Azar, KWang, G Wong, F Gutierrez, T Rappaport, in Communications (ICC), 2013 IEEE International Conference on. $28 \mathrm{GHz}$ millimeter wave cellular communication measurements for reflection and penetration loss in and around buildings in New York city (IEEE, Budapest, 2013), pp. 5163-5167. doi:10.1109/ICC.2013.6655403

4. 5G Candidate Band Study: Study on the Suitability of Potential Candidate Frequency Bands above $6 \mathrm{GHz}$ for Future $5 \mathrm{G}$ Mobile Broadband Systems (2015). http://stakeholders.ofcom.org.uk/binaries/consultations/above6ghz/qa-report.pdf

5. M Samimi, K Wang, Y Azar, G Wong, R Mayzus, H Zhao, J Schulz, S Sun, F Gutierrez, T Rappaport, in Vehicular Technology Conference (VTC Spring), 2013 IEEE 77th. $28 \mathrm{GHz}$ Angle of Arrival and Angle of Departure Analysis for Outdoor Cellular Communications Using Steerable Beam Antennas in New York City (IEEE, Dresden, 2013), pp. 1-6. ISSN:1550-2252, doi:10.1109/NTCSpring.2013.6691812

6. S Hur, Y-J Cho, J Lee, N-G Kang, J Park, H Benn, in Communications and Networking (BlackSeaCom), 2014 IEEE International Black Sea Conference on. Synchronous channel sounder using horn antenna and indoor measurements on $28 \mathrm{GHz}$ (IEEE, Odessa, 2014), pp. 83-87. doi:10.1109/BlackSeaCom.2014.6849010

7. J Medbo, K Borner, K Haneda, V Hovinen, T Imai, J Jarvelainen, T Jamsa, A Karttunen, K Kusume, J Kyrolainen, P Kyosti, J Meinila, V Nurmela, L Raschkowski, A Roivainen, J Ylitalo, in Antennas and Propagation (EUCAP), 2014 8th European Conference on. Channel modelling for the fifth generation mobile communications (IEEE, The Hague, 2014), pp. 219-223. doi:10.1109/EuCAP.2014.6901730

8. K Guan, B Ai, A Fricke, D He, Z Zhong, D Matolak, T Kuerner (2016). Excess Propagation Loss of Semi-Closed Obstacles for Inter/Intra-Device Comtlmunications in the Millimeter-Wave Range Journal title: Journal of Infrared, Millimeter and Terahertz Waves ISSN (Print) 1866-6892 - ISSN (Online) 1866-6906, doi:10.1007/s10762-016-0251-3, http://link.springer. com/article/10.1007\%2Fs10762-016-0251-3

9. K Haneda, Channel models and beamforming at millimeter-wave frequency bands. IEICE Trans. Commun. E98-B(5), 755-772 (2015)

10. S Salous, V Degli-Esposti, M Nekovee, S Hur, in Antennas and Propagation (EuCAP), 2015 9th European Conference on. Review of Millimeter-wave Propagation Characterization and Modelling Towards 5G Systems (IEEE, Lisbon, 2015)

11. A Sulyman, A Nassar, M Samimi, G MacCartney, TRappaport, A Alsanie, Radio propagation path loss models for $5 \mathrm{G}$ cellular networks in the 28 $\mathrm{GHZ}$ and $38 \mathrm{GHZ}$ millimeter-wave bands. IEEE Commun. Mag. 52(9), 78-86 (2014)

12. A Polydorou, G Stratakos, C Capsalis, N Uzunoglu, Comparative study of millimeter wave propagation at $30 \mathrm{GHz}$ and $60 \mathrm{GHz}$ in indoor environment. Int. J. Infrared Millimeter Waves. 16(10), 1845-1862 (1995)

13. D Beauvarlet, $\mathrm{K}$ Virga, Measured characteristics of $30-\mathrm{GHz}$ indoor propagation channels with low-profile directional antennas. IEEE Antennas Wirel. Propag. Lett. 1(1), 87-90 (2002)

14. K Guan, Z Zhong, B Ai, T Kurner, Empirical models for extra propagation loss of train stations on high-speed railway. IEEE Trans. Antennas Propag. 62(3), 1395-1408 (2014)

15. K Guan, Z Zhong, B Ai, T Kurner, Propagation measurements and modeling of crossing bridges on high-speed railway at $930 \mathrm{mhz}$. IEEE Trans. Veh. Technol. 63(2), 502-517 (2014)

16. I Cuiñas, M Garcia Sanchez, Wide-band measurements of nondeterministic effects on the BRAN indoor radio channel. IEEE Trans. Veh. Technol. 53(4), 1167-1175 (2004)

17. K Guan, B Ai, M Liso, R Geise, A Moller, d Zhong, T Kuerner, On the influence of scattering from traffic signs in vehicle-to-x communications. IEEE Trans. Veh. Technol. PP(99), 1 (2015)
18. J Andersen, J Nielsen, G Pedersen, G Bauch, M Herdin, Room electromagnetics. IEEE Antennas Propag. Mag. 49(2), 27-33 (2007)

19. A Bamba, W Joseph, JB Andersen, E Tanghe, G Vermeeren, D Plets, JO Nielsen, L Martens, Experimental assessment of specific absorption rate using room electromagnetics. IEEE Trans. Electromagn. Compat. 54(4), 747-757 (2012). doi:10.1109/TEMC.2012.2189572

20. J Nielsen, J Andersen, G Pedersen, M Pelosi, in Vehicular Technology Conference (VTC Fall), 2011 IEEE. On polarization and frequency dependence of diffuse indoor propagation (IEEE, San Francisco, CA, 2011), pp. 1-5. doi:10.1109/NETECF.2011.6092906

21. J Fuhl, J-P Rossi, E Bonek, High-resolution 3-D direction-of-arrival determination for urban mobile radio. IEEE Trans. Antennas Propag. 45(4), 672-682 (1997)

22. P Stoica, RL Moses, Introduction to Spectral Analysis. (Prentice-Hall, Cliffs, NJ, 1997)

23. A Kuchar, J-P Rossi, E Bonek, Directional macro-cell channel characterization from urban measurements. IEEE Trans. Antennas Propag. 48(2), 137-146 (2000)

24. C Gentile, AJ Braga, A Kik, A comprehensive evaluation of joint range and angle estimation in indoor ultrawideband location systems. EURASIP J. Wirel. Commun. Netw. 2008, 36 (2008)

\section{Submit your manuscript to a SpringerOpen ${ }^{\circ}$ journal and benefit from:}

- Convenient online submission

Rigorous peer review

- Immediate publication on acceptance

- Open access: articles freely available online

- High visibility within the field

- Retaining the copyright to your article

Submit your next manuscript at $\gg$ springeropen.com 